\title{
Pratiques alimentaires des diabétiques. Étude de quelques cas à Oran (Algérie)
}

Food practices of the diabetics. Study of some cases in Oran (Algeria)

\section{Ouassila Salemi}

\section{(2) OpenEdition}

\section{Journals}

\section{Édition électronique}

URL : http://journals.openedition.org/economierurale/2816

DOI : $10.4000 /$ economierurale.2816

ISSN : 2105-2581

\section{Éditeur}

Société Française d'Économie Rurale (SFER)

\section{Édition imprimée}

Date de publication : 1 septembre 2010

Pagination : 80-95

ISSN : 0013-0559

\section{Référence électronique}

Ouassila Salemi, «Pratiques alimentaires des diabétiques. Étude de quelques cas à Oran (Algérie) », Économie rurale [En ligne], 318-319 | juillet-octobre 2010, mis en ligne le 01 octobre 2012, consulté le 23 avril 2019. URL : http://journals.openedition.org/economierurale/2816 ; DOI : 10.4000/ economierurale.2816 


\section{Pratiques alimentaires des diabétiques Étude de quelques cas à Oran (Algérie)}

Ouassila SALEMI • Groupe de recherche en anthropologie de la santé (GRAS)

Université d'Oran, Algérie

\section{Introduction}

e diabète ${ }^{1}$ est l'une des causes de décès les

-plus importants dans le monde. Il prend des proportions alarmantes et suscite de vives inquiétudes chez les praticiens en charge de cette maladie. Selon l'Organisation mondiale de la santé (OMS), il y a plus de 180 millions de diabétiques dans le monde. Un chiffre d'autant plus effarant qu'on prévoit le double à l'horizon de 2030. Alors qu'ils étaient très rares il y a encore vingt ans, $63 \%$ des diabétiques vivent dans les pays en développement. L'OMS estime que le taux de mortalité dû au diabète va augmenter de $50 \%$ durant la prochaine décade si aucune mesure urgente n'est prise. Véritable épidémie, le diabète est responsable de plus de décès que le VIH, il tue une personne toutes les dix secondes.

En Algérie, le diabète constitue l'un des problèmes majeurs de santé publique, en particulier le diabète de type 2 et ce en raison de l'explosion de son incidence (Kourta, 2006). Sur une population estimée à 32 millions d'habitants, et selon la Fédération algérienne des associations des diabétiques, le nombre de diabétiques a atteint le chiffre de 2 millions dont $21 \%$ d'insulinodépendants. Selon la Société algérienne de diabétologie, $90 \%$ de la population des diabétiques présentent le diabète de type 2 et $10 \%$ de type 1 (Hadjiat, 2006). L'Algérie traverse

1. Le diabète est une maladie caractérisée par une hyperglycémie (hausse du taux de glycémie dans le sang) chronique résultant d'un défaut de sécrétion d'insuline (hormone produite par le pancréas) ou de son action ou alors de ces deux anomalies associées. Il se présente principalement sous deux formes : le diabète de type 1 : (ou diabète juvénile) et le diabète de type 2 :(ou diabète obèse). depuis deux décennies une transition sanitaire. En effet, le vieillissement progressif de la population s'est accompagné d'une augmentation des pathologies chroniques non transmissibles. Une enquête nationale, nommée Transition and Health Impact in North Africa (TAHINA) a été menée dans le cadre global d'un projet de recherche sur la transition épidémiologique et son impact sur la santé en Afrique du nord. Elle a été réalisée en 2005 par l'Institut national de santé publique d'Alger (INSP) en collaboration avec l'Union européenne dans 16 wilayas (départements). Selon cette étude, le diabète est le deuxième état morbide le plus fréquent $(8,78 \%)$ après l'hypertension artérielle $(16,23)$. Il touche $12,21 \%$ d'Algériens dont une prédominance féminine de $12,54 \%$. Le diabète est plus fréquent en milieu urbain, il est de l'ordre de 10,15\% contre $6,40 \%$ en milieu rural.

Durant notre présence, aussi bien au service de médecine interne qu'au centre de santé de Maraval, les femmes étaient plus nombreuses à consulter. En outre, le service de diabétologie du centre hospitalouniversitaire d'Oran, a confirmé que les femmes sont les plus exposées au diabète. Les principales raisons en cause sont liées à l'obésité qui influe à $70 \%$ sur la santé des femmes et les expose aux complications du diabète, ensuite les facteurs liés aux troubles psychiques (Kourta, 2008). Cette prédominance féminine n'est pas spécifique à l'Algérie. En effet, une série d'aspects sociaux, culturels et économiques du fardeau discriminatoire du diabète pèse sur les femmes dans quasiment toutes les régions du monde (Henrichs, 2009). En outre, selon l'OMS, près de la moitié des décès impu- 
tables au diabète surviennent chez des personnes de moins de 70 ans et $55 \%$ des personnes qui meurent de la maladie sont des femmes.

Dans la perspective médicale de la prise en charge du diabète, le respect du régime alimentaire revêt une dimension importante. En effet, l'administration du traitement médical, qu'il s'agisse de l'insuline ou d'antidiabétiques oraux est tributaire d'une alimentation hypocalorique, réduite en lipides saturés et en sucres simples. L'approche médicale, centrée sur l'équilibre de la glycémie et sur la prévention des complications du diabète rejoint dans son optique, l'éducation nutritionnelle conventionnelle dont l'objectif est la modification des comportements alimentaires. Elle est basée sur l'hypothèse selon laquelle l'ignorance et le manque de connaissances diététiques sont à incriminer dans les mauvaises conduites alimentaires et que l'acquisition d'un savoir et d'un savoir-faire est à même d'aboutir à une amélioration de la situation nutritionnelle (Calandre, 2002). En effet l'information sur les risques encourus à la suite du non respect du régime alimentaire n'était pas suffisante pour changer les pratiques des diabétiques. Il nous semble que cette attitude qualifiée d' « irrationnelle » par le corps médical doit nous inciter à rechercher les dimensions sociales qui imprègnent profondément la vie quotidienne. Or bien souvent, les médecins prennent peu ou prou en considération la dynamique socio-alimentaire. Le faible impact des campagnes de prévention et de sensibilisation à une nourriture diététique montre bien qu'au-delà de sa dimension bio-nutritionnelle, l'alimentation intègre d'autres préoccupations d'ordre socio culturel (Calandre, op. cit.). La limite de l'éducation nutritionnelle est qu'elle met en position dominante les critères de santé et de nutrition, en éclipsant les autres fonctions de l'alimentation, hédonique, sociale et culturelle. Or l'alimentation n'est pas seulement la satisfaction d'un besoin physiologique, elle est un acte complexe qui renvoie à des réalités psychologiques, sociales et culturelles
(Lahlou, 1988). L'alimentation est une production sociale et un système de comportements et de représentations de la vie sociale (Garabuau-Moussaoui, 2002) : «Les hommes ne mangent pas des nutriments mais des aliments cuisinés, combinés entre eux au sein de préparations culinaires (...) selon un protocole fortement socialisé » (Poulain, 2002).

Selon l'enquête TAHINA, «les Algériens mangent mal », la consommation alimentaire quotidienne ne respecte pas les recommandations internationales de santé (Kourta, 2006). Elle est jugée faible en fruits et légumes $(0,6$ fruit par jour au lieu des deux portions recommandées et 0,8 légume par jour au lieu des trois portions recommandées). En revanche, la consommation des produits gras et sucrés est en deçà des normes (2,7 portions-jour contre une portion recommandée).

Le regard socio-anthropologique, qui tente de comprendre la complexité de l'objet aliment, s'inscrit dans une logique de refus du jugement de valeur sur les pratiques alimentaires (Mebtoul, 2007). Il s'agit pour nous, de questionner les logiques sociales déployées par les diabétiques à l'égard de l'alimentation, le sens attribué au régime et les différentes contraintes auxquelles ils font face. Il nous semble que la compréhension des sens donnés à l'aliment et au régime est importante avant de décréter la transformation autoritaire des comportements alimentaires. Car comme le proclamait déjà Margareth Mead dès 1945 : "Avant de chercher à savoir comment changer les habitudes alimentaires [il faut tout d'abord] comprendre ce que manger veut dire ». Françoise Héritier-Augé (1985) avait écrit : "L'autre, c'est d'abord celui qui ne mange pas comme soi ». L'alimentation apparaît ainsi comme le socle à partir duquel se développent aussi bien les identités individuelles que collectives. L'alimentation en tant que fait social constitue un ensemble de représentations, savoirs et pratiques qui s'affirment dans ses différences par rapport à 
d'autres systèmes alimentaires (Suremain et al., 2006).

En Algérie, l'alimentation constitue le point nodal de différents regroupements qui ont lieu à l'intérieur des espaces domestiques ou en dehors, lors de repas familiaux habituels ou au cours d'occasions comme les mariages, les naissances, etc. Des regroupements plus importants de populations venues des alentours ou de régions plus éloignées se réunissent dans les waâda ${ }^{2}$. Elles viennent célébrer en communion et dans la liesse, le plus souvent autour d'un grand couscous, l'ancêtre commun, ou rendre hommage à un saint homme vénéré auquel est dédié un mausolée. Se retrouver pour manger ne se limite pas aux événements heureux. Les funérailles sont aussi des occasions pour partager la nourriture, exprimer sa compassion, renforcer les liens familiaux et communautaires. L'histoire alimentaire algérienne témoigne d'une très ancienne expérience agronomique et d'une riche tradition culinaire. Malgré l'avènement de l'industrie agroalimentaire, les Algériens restent très attachés aux plats traditionnels comme le couscous, le berkoukes ${ }^{3}$, la chakhchoukha ${ }^{4}$ et les différentes soupes et pâtes, qui sont toujours appréciés aussi bien dans les compagnes que dans les villes. Les plats autochtones associés aux apports extérieurs constituent la cuisine algérienne actuelle, changeante d'une région à une autre, souvent par de légères variantes et parfois de façon radicale (Bouayad, 1978). Les gâteaux ne sont pas en reste, makrout ${ }^{5}$, griwèche $e^{6}$ et autres douceurs

2. Les waâda ou zerda constituent des repas festifs occasionnels pour célébrer les saints spécifiques à chaque région ou seulement partager équitablement la viande issue de l'abattage de moutons ou de bœufs. 3. Gros grains de semoule roulée, cuits dans une sauce faite de légumes et de viande.

4. Lambeaux de pâte feuilletée arrosés de sauce de légumes et de viande

5. Losanges de semoule farcis à la patte de dattes, frits dans l'huile et enrobés de miel.

6. Oreillettes de farine et de beurre, frites dans l'huile, enrobées de miel et parfumées aux grains de sésame. sont présentes pour agrémenter les $m e i d a^{7}$ autour desquelles membres de la famille et hôtes, dégustent avec du thé ou du café. Nos pratiques culinaires imposent le partage avec les membres de la famille (Mebtoul, op. cit.). En effet, le plat collectif est prédominant dans les foyers algériens $(52,07 \%$ des Algériens mangent dans le plat commun). En outre, tous les repas sont pris à domicile pour plus de $93 \%$ des cas et les trois principaux repas sont pris en famille.

Afin de répondre à notre problématique, nous avons privilégié l'approche qualitative. Notre posture socio-anthropologique qui s'inscrit dans l'altérité a consisté à restituer les propos de l'autre par la recherche de la qualité des discours émis par les interviewés. Elle est basée sur des entretiens individuels approfondis, répétés et de longue durée (entre une heure et demi et deux heures environ), et l'observation fine et détaillée des différents espaces investis. Ces deux techniques jumelées nous paraissent les mieux adaptées afin de «relever les discours et les situations permettant d'accéder aux croyances, aux représentations, aux pratiques et aux institutions qui donnent sens à une société » (Fassin, 1990). Nos investigations se sont déroulées en premier lieu au service de médecine interne de la nouvelle structure hospitalière $1^{\text {er }}$ novembre d'Oran. Ce choix se justifie par le fait qu'il est un service de consultation et d'hospitalisation de malades diabétiques. En outre, la présence en son sein d'un allié a constitué une opportunité. En effet, l'obtention d'une autorisation d'enquête a été facilitée et la circulation à l'intérieur du service sans porter de blouse ainsi que la possibilité de s'entretenir en aparté avec les patients ont constitué des atouts pour le bon déroulement de l'enquête. Nous avons pu avoir accès aux dossiers des malades et nous nous sommes entretenus avec huit d'entre eux : cinq femmes et trois hommes, âgés de 25 à 52 ans. Dans un souci de diversification,

7. Table basse. 
nous avons opté pour un second lieu, le centre de santé de Maraval situé dans la même ville. Cette structure de santé compte parmi les unités sanitaires de base. Ces dernières se trouvent être la première destination des malades $(52,09 \%$ des cas y ont recours, selon TAHINA). Le médecin généraliste y exerçant est notre informateur privilégié. Il est chargé du suivi des malades chroniques, principalement des diabétiques, pour certains de très longue date. Nous avons pu assister aux consultations, rencontrer les malades, pour ensuite prendre rendez-vous pour des entretiens à domicile. Nous avons interviewé huit femmes au foyer âgées entre 43 et 65 ans, différentes dans leurs caractéristiques sociodémographiques ( $c f$. l'annexe). Les entretiens se sont déroulés pendant le mois de Ramadhan. Les propos des enquêtés ont été recueillis sur un enregistreur numérique. Le matériel sonore a été traduit de l'arabe dialectal et saisi en français.

Trois parties structurent cet article. Les contraintes économiques seront d'abord évoquées. En effet, nos interviewés liaient les difficultés de suivre le régime préconisé à la précarité de leur situation. Puis, nous montrerons que les pratiques alimentaires sont révélatrices de la nature des relations existant au sein des familles ainsi que du statut du diabète dans la société. Enfin, nous verrons, comment certaines habitudes et traditions culinaires ont un impact sur les pratiques alimentaires des diabétiques, et comment l'alimentation renvoie à des univers de sens aussi variés que le plaisir, la convivialité et la commensalité.

\section{Les contraintes économiques}

Le diabète devient de plus en plus une maladie des pauvres (Hamadachi, 2009). Les difficultés économiques suivies de la dévaluation du dinar algérien (DA) ont provoqué une augmentation significative du prix des aliments de base, notamment des céréales. L'étude réalisée en 2006 par le Centre national d'études et d'analyses pour la population et le développement (CENEAP) a révélé que $11,1 \%$ des ménages algériens sont pauvres, 15,2\% vivent en milieu rural et $8 \%$ dans les centres urbains ${ }^{8}$.

La relation entre le niveau des ressources financières et la consommation est apparue déterminante dans les conduites alimentaires des diabétiques interviewés. En effet, dans un contexte marqué par la cherté des produits de consommation, la composition du panier de provisions reste tributaire des fluctuations des prix du marché. Cette situation s'accentue pendant la période de Ramadhan où on assiste à une véritable flambée des prix. Devant cet état de fait, les femmes, actrices sociales incontournables dans les pratiques culinaires (Mebtoul, op. cit.) mettent en branle une imagination créatrice afin de concocter le fameux ftour ${ }^{9}$ du Ramadhan. Houria, 65 ans, est diabétique depuis douze ans et mère de deux filles et trois garçons. Son mari est retraité. Deux de ses fils sont partis en France. Son troisième fils ne travaille pas, il vit avec sa femme et son enfant. À peine avions-nous abordé le thème du régime qu'elle s'exclama :

«On ne va pas se mentir, ce n'est ni facile, ni évident de faire le régime! Ils nous disent de manger ceci, de ne pas manger cela, mais nous, on n'a pas... il n'y a pas! On n'est pas en Europe, là-bas oui, il y a de tout, ici non. Que te dire sinon qu'on espère que Dieu soit avec nous. »

Le régime alimentaire apparaît ainsi comme incongru devant un budget extrêmement serré. Il n'est pas question pour Houria de penser au régime dans de telles conditions :

«Quand mon fils m'a lu la feuille que lui a remise le médecin, où étaient mentionnés les

8. Le seuil de pauvreté général étant de 5,7\%, le pourcentage restant représente les couches intermédiaires et aisées.

9. Le ftour est le repas pris à la rupture du jeûne du mois de Ramadhan. 
aliments que je dois prendre pour mon régime, je ne pouvais qu'en rire. Le régime demande des moyens que je n'ai pas. Mon fils ne travaille pas. La retraite de mon mari ne peut pas subvenir à tous nos besoins. Nous nous ingénions, ma belle fille et moi à préparer des plats qui soient agréables et suffisants pour tout le monde, surtout en cette période de Ramadhan. Alors pour ce qui est du régime, c'est une autre paire de manche. Manger des fruits comme les pommes, de la viande grillée, des légumes, c'est vraiment impossible... Impossible... »

Ainsi le régime n'est pas envisagé à cause de la restriction budgétaire. Le plus important est de concilier menu et prix du marché. Avec la retraite de son mari qui est de l'ordre de 10000 DA (avoisinant les 100 euros), Houria se tourne vers les denrées alimentaires les moins chères à l'exemple du riz et de la pomme de terre. Mais cette dernière est devenue, elle aussi inabordable:

«Le médecin m'a encore dit hier de faire le régime, mais avec quoi ?avec $10000 \mathrm{DA}$ comment faire... Est-ce qu'avec 10000 DA, on peut acheter les pommes, la viande de bouf, le poulet, etc. Maintenant, on est obligé d'acheter du riz et de la pomme de terre, que te dire sinon que nous vivons "la situation du pauvre" et même la pomme de terre qui était le légume du pauvre, avec laquelle on arrivait à faire la cuisine sans trop se casser la tête, eh bien! Elle est devenue un luxe, son prix avoisine les 100 DA ! Tu te rends compte elle est devenue aussi chère que les fruits sinon plus ! On nous dit de faire le régime mais il faut voir comment on vit!»

Saliha a 39 ans. Elle est diabétique depuis trois ans. Son mari est instituteur. Elle a cinq enfants. Elle aussi, invoque des contraintes d'argent comme un obstacle dans le suivi de son régime:

«Il ne suffit pas de dire au malade de faire le régime, ce n'est pas aussi facile! Le diabétique a besoin de manger, de bien manger et de manger de bonnes choses, comme les pommes par exemple. Et cela nécessite de l'argent bien sûr! La dernière fois j'étais malade, le médecin, m'a dit que j'ai une carence en calcium et en vitamines. Il $m$ 'a dit que ce que je mange est pauvre en vitamines. Mais comment faire! »

La paie de son mari étant insuffisante, Saliha a trouvé quelques astuces pour pallier le manque d'argent :

« Je fais la "hrira” pour deux jours, je ne jette pas les restes, je mets au frigo et je garde pour les enfants. Le Ramadhan est synonyme de dépenses. La paye de mon mari est de 24000 DA et avec le mariage prochain de ma fille, je suis en train de préparer son trousseau. J'ai vendu mes bijoux en or et mon mari n'est pas au courant. Tu sais comment je fais pour me débrouiller? Eh bien, je mets des pièces de monnaie, au fur et à mesure, dans la tirelire et quand il y a assez d'argent, j'achète ce qui manque. J'achète à l'insu de mon mari. Lui, il achète le gros, la pomme de terre, les légumes... Des fois avec 300 DA, j'achète quelques épices, du sel, du vinaigre. Par exemple, ce matin, en emmenant ma fille à l'école, j'ai acheté le savon à 50 DA. Si je lui dis, il me répondra, qu'il n'a pas. Lui, il se contente de faire le marché et il me dit de me débrouiller, alors que les diabétiques ont besoin de bien manger et de prendre des vitamines. »

Djamila a 51 ans, cadette de quatre filles et de deux garçons, elle est célibataire et vit seule. Ancienne couturière, elle est diabétique depuis trente ans. Ne pouvant pas travailler depuis l'opération faite sur sa main, elle ne vit que de la retraite de sa mère qui est de l'ordre de 1000 DA (10 euros). Elle ne perçoit plus la pension chômage estimée à 3000 DA :

«Le régime demande des moyens importants. Avant quand je travaillais, ça ne posait pas de problème, j'achetais avec mon propre argent. J'avais la possibilité 
d'acheter et quoi acheter. Quand tu as ton propre argent, tu peux dépenser comme tu veux, mais dans le cas contraire, tu restes enchaînée. Tu ne peux rien faire et c'est vraiment difficile. La retraite des vieux n'est que de 1000 DA. Je t'assure que je ne vis que de 1000 DA et encore, il y a quelque temps, je ne recevais que 800 DA... C'est la retraite de ma mère, mon père n'en a pas, il n'a jamais vraiment travaillé. Les gens croient que je touche 10000 DA. Quand je leur ai montré le papier ou était inscrit le chiffre, ils ne croyaient pas leurs yeux, ils ajoutaient comment fais-tu pour vivre? Tu sais, bien que je sois dans le besoin, je ne demande jamais l'aumône aux gens. S'ils veulent bien me donner, j'accepte, sinon je suis comme tu me vois. Avant je n'avais pas trop de problème pour suivre mon régime, mais maintenant, ce n'est pas moi qui parle, c'est celle-là... (Elle montre la fiche de paiement du mois courant), je n'ai rien reçu. Je ne touche plus l'argent $d u$ chômage. Je suis en train de courir à droite et à gauche pour régler le problème. Dismoi comment vais-je faire pour payer l'eau, l'électricité, le gaz. Et si j'arrive à les payer, que me resterait-il pour acheter le marché, je suis vraiment entrain d'y penser... il n'y a rien, que veux-tu cuisiner, plutôt que peuxtu cuisiner, alors l'essentiel c'est de trouver quelque chose à manger, n'importe quoi!»

Pendant le mois de Ramadhan, période durant laquelle l'entretien a été réalisé, Djamila est invitée chez sa sœur aînée pour manger chez elle :

«Pendant ce mois de Ramadhan, je vais manger chez ma grande sœur, elle n'habite pas très loin. Elle s'est toujours occupée de moi, surtout quand je tombe malade. Moi aussi, j'ai toujours été à ses côtés, lors de son opération, j'étais à son chevet. Elle et son mari ont insisté, ils savent très bien dans quelle situation je suis et je t'avoue que je n'ai pas le choix. Mais cela ne veut pas dire que je ne cuisine pas du tout. Je prépare quand même quelque chose, il se pourrait que mon frère vienne chez moi, donc il doit $y$ avoir de quoi manger, de toute façon, s'il ne vient pas, je garde la nourriture pour le shour $^{10}$.»

Ces illustrations montrent bien la difficulté de concevoir le régime devant les contraintes liées à la cherté de la vie. Ainsi, « la conformité aux normes nutritionnelles ne fait pas partie des préoccupations premières, qui relèvent de la gestion d'une forme de pénurie, du poids des tâches domestiques et d'une faible intégration sociale » (Régnier, 2009).

Il n'est pas inutile de rappeler que le diabète et ses conséquences, en termes de mortalité et de morbidité, particulièrement en ce qui concerne le développement des complications, frappent en premier lieu les membres des communautés les plus vulnérables, principalement, les personnes à faible niveau socio-économique, réactivant ainsi la question récurrente et préoccupante des inégalités de santé (Imbert, op. cit.). Ainsi, les disparités financières pour avoir accès à des aliments sains expliquent pourquoi l'incidence du diabète et de l'obésité se trouve parmi les populations les moins favorisées, à fort taux de chômage (Bihr et al., 2000). Mais les facteurs économiques et les limites des revenus ne permettent pas à eux seuls d'expliquer le comportement des diabétiques. Ils s'accompagnent d'autres considérations liées aux rapports sociaux de sexe au sein de la famille, dans l'organisation sociale et du statut du diabète dans la société.

\section{Relations familiales et statut du diabète}

Manger ensemble apprend à partager une culture, à manier un système de signes et implique des conduites normées et interdépendantes de prise en compte d'autrui

10. Le shour est le repas pris tard dans la soirée pour pouvoir tenir la journée pendant le mois de Ramadhan. 
(Rivière, 1995). Ainsi les pratiques alimentaires sont révélatrices de la nature des rapports sociaux qui se tissent au sein des espaces domestiques. Camille LacosteDujardin (1996), dans son ouvrage traitant de la maternité et du patriarcat au Maghreb, a développé une analyse très pertinente des relations familiales. Elle a mis en exergue la particularité de la relation qui lie le fils à sa mère :

«Le mariage du fils a donc introduit une tierce personne aux côtés de la dyade mèrefils : une jeune femme... La mère gagne incontestablement en pouvoir : à sa fonction maternelle simple s'ajoute donc désormais celle d'une responsable de l'entreprise domestique, organisant et dirigeant le travail des personnes qui sont à présent placées sous ses ordres... En fait, à l'influence maintenue entière sur son fils vient s'ajouter une domination réelle sur la nouvelle venue, où les liens affectifs n'ont au moins au début, que fort peu de part, quand ils ne sont pas chargés d'hostilité et de jalousie latente. Quand à la nouvelle jeune femme, elle ne se hasarde guère, à son arrivée, à tenter d'acquérir quelque pouvoir. Préparée dès l'enfance, et par une femme, sa mère, à l'école de la soumission et du travail domestique de la maisonnée, de peur d'encourir le risque d'une répudiation qui compromettrait ses chances d'accéder à son tour au seul statut possible et gratifiant pour une femme : celui de mère de fils."

Camille Lacoste-Dujardin montre bien comment la femme n'accède au statut que la société lui reconnait, qu'en se mariant et en devenant mère et de garçons de surcroit. Cette tradition de la femme mère-avanttout est intériorisée par la femme elle-même selon les préceptes de l'idéologie patriarcale basée sur la domination masculine. Nos pratiques alimentaires sont révélatrices de la prégnance du fonctionnement de ce modèle traditionnel basé sur les rapports sociaux de sexe. Les hommes mangeant seuls, ou alors il y a un ordre des repas : celui des hommes précédant celui des femmes (Lacoste Dujardin, 1996). Le régime alimentaire du diabétique, produisant des modifications dans les pratiques culinaires socialisées au sein de l'espace familial, peut être ainsi producteur de tensions entre les membres de la famille. Il semble ainsi difficile de concilier le régime individuel avec un repas conçu pour toute la famille, surtout quand la personne concernée par le régime est une femme, socialement dominée. C'est le cas de Kheira, 56 ans, mère de cinq garçons et de deux filles et diabétique depuis dix-neuf ans. Hypertendue, elle doit ainsi suivre un régime demi-sel. Elle disait :

«Au moment de servir, les garçons qui mangent avec leur père ne sont pas satisfaits. Ils émettent des commentaires, ils veulent que le repas soit impeccable, ils ne veulent pas resaler, ils disent qu'ils ne sont pas malades, alors je dis à ma fille de ne pas prendre en considération mon régime pour éviter les problèmes. »

Sa fille l'interrompt : "Quand ils rouspètent, je ne me tais pas. Je leur dis que le régime est important. D'ailleurs quand je mange avec ma mère je ne resale pas. ».

Et sa mère d'ajouter : «C'est surtout mon mari à qui ça ne plaît pas, les garçons sont plutôt compréhensifs, surtout l'aîné. D'ailleurs c'est lui (l'aîné), malgré le fait qu'il n'a pas de travail fixe qui m'achète les médicaments non remboursables que je prends pour mon traitement contre les champignons. »

Ainsi les relations familiales structurent les pratiques alimentaires. Ces dernières basées sur des repas conçus pour toute la famille piègent souvent le régime alimentaire centré sur la personne malade (Mebtoul, op. cit.) et tiennent compte aussi des rapports sociaux de sexe. Même si de plus en plus de jeunes mariés vivent en dehors du cocon familial, de nombreuses mariées se plaignent de l'ingérence de la belle-mère dans la vie du «couple ». Benabed (2008), dans son étude sur les 
couples stériles, a montré l'emprise de la belle mère dans la relation conjugale et la pression qu'elle exerce sur le couple dès que l'enfant tarde à venir. Cette dernière se manifeste dans son rôle de contrôleuse de la vie du couple s'exprimant par la violence symbolique des mots. L'entretien avec Amina, 25 ans, universitaire, mère de trois filles, diabétique depuis quatre ans, est révélateur de cette emprise. Contrainte de cacher sa maladie à sa belle-mère, elle déclare :

«Il n'y a que ma famille (mes parents, mes frères et sours) et mon mari qui sont au courant de mon diabète. Déjà que ma belle mère voit mal que je sois asthmatique, et pour cela elle me traite de messdoura ${ }^{l 1}$. Si elle apprenait que je suis diabétique ce serait pire!»

La belle-mère continue à jouer un rôle essentiel dans l'espace familial (Mebtoul, 2000) même si elle n'habite pas sous le même toit que son fils. La belle-mère d'Amina qui n'a pas eu son mot à dire pour le mariage de son fils unique, semble constituer une menace pour la stabilité du couple : «Elle n'a jamais vraiment accepté notre mariage, mon mari l'a mise devant le fait accompli. Naïve comme je suis, je leur ai dit que j'étais asthmatique. Depuis elle ne rate pas une occasion pour me le rappeler, surtout quand elle vient chez moi et qu'elle me trouve souffrante. D'ailleurs, elle n'arrête pas de répéter que son fils devrait épouser une femme en très bonne santé, alors si par malheur elle apprenait que je suis diabétique, j'appréhende le pire... À l'annonce de la maladie, je me suis dit que c'était fini pour moi, que ma vie était finie... Mais grâce au soutien de ma famille et de mon mari, j'ai pu remonter la pente. Mon mari m'aide beaucoup, il m'encourage à bien prendre soin de moi, pour moi pour lui et pour les enfants. »

11. Ce mot veut dire littéralement atteinte à la poitrine. Il est fort stigmatisant car il fait allusion à la tuberculose.
Hafida est âgée de 33 ans, elle est diabétique depuis trois ans. L'histoire de sa maladie dénote le rapport conflictuel entretenu avec la belle-mère, au point de lier la survenue de sa maladie à cette dernière. À ce propos, elle disait :

«Même si je sais que le diabète me vient de ma famille, mes parents, mes trois frères et deux sœurs sont diabétiques, mais, ce sont les problèmes et les conflits avec ma belle mère qui ont provoqué mon diabète. Il ne se passait pas un jour sans qu'il y ait de querelles, mais j'étais obligée de supporter parce que mon mari n'a pas les moyens de louer dehors jusqu'au jour où on a été tout simplement expulsés de la maison et nos affaires jetées dehors. Heureusement que j'ai une sæur ici qui a accepté de mettre nos affaires chez elle et de nous héberger provisoirement, le temps qu'on trouve une solution. Je ne sais vraiment pas quoi faire ni comment faire, pour le moment je compte sur la patience de ma sour et de son mari et sur leur générosité... Quand j'étais chez ma belle mère, c'est elle qui décidait du menu, je faisais la cuisine pour toute la famille. Il n'était pas question de suggérer quoi que ce soit, d'ailleurs le fait que je sois diabétique n'a rien changé à la situation. Ma belle mère est autoritaire et ne veut rien savoir. On devait manger et se taire!»

Ainsi la gestion profane du diabète ne se fait pas sans relation avec le fonctionnement quotidien des différents espaces sociaux (famille, lieu de travail, etc.). Il est intéressant d'observer que le diabétique met en avant une perspective qui octroie la primauté aux relations sociales dominantes et prend ainsi en compte les jugements des autres et les interdits sociaux au cœur de la société (Mebtoul, 2003) sans s'embarrasser de la rationalité de la norme médicale :

«Quand je suis invitée chez des gens, je me comporte le plus normalement du monde. Je prends un gâteau ou deux et j'augmente la dose d'insuline. Même au travail, personne n'est au courant. Pour mon insuline, je me 
pique aux toilettes et c'est mieux comme ça! » (Amina)

C'est aussi, le cas de Saliha, 45 ans, cinq enfants, diabétique depuis cinq ans. Lors du mariage de sa fille, elle a du passer la nuit hors de chez elle. Elle n'a pas emporté ses médicaments avec elle et elle a mangé comme tout le monde. Elle affirmait :

«Je suis partie au sud pour le mariage de ma fille et je suis restée deux jours sans faire mes injections. Je ne voulais pas la faire devant les gens, tu sais les gens comment ils sont... En plus je n'ai pas trouvé un endroit où la faire loin des regards. Je ne voulais pas qu'on s'apitoie sur mon sort et entendre dire "la pauvre elle est malade». Je l'ai donc laissé dans mon sac et je suis revenue avec, mais j'ai souffert après. Je ne te cacherais pas aussi que j'ai pensé aussi à ma fille mariée et à mes autres filles. Tout le monde sait maintenant que le diabète est héréditaire, je ne veux pas que l'on insinue quoi que ce soit à ma fille qui venait de se marier. Je ne voudrais pas aussi compromettre les chances de mariage de mes autres filles, je préfère que tout ça reste dans le secret. »

Cette discrétion liée au mariage est apparue aussi dans le discours de Djamila : «À part mes sœurs, personne ne sait que je suis diabétique, je ne fréquente pas les gens, je ne vais pas chez les voisins. Je n'aime pas divulguer mes secrets. Les gens parlent trop. Je ne veux pas qu'ils le sachent, ils vont commencer à dire, elle a ceci, elle a cela, elle a fait ceci, elle a fait cela. Je ne veux rien de tout cela. Je ne veux pas en parler. En plus, si quelqu'un se présentait pour demander ma main, je ne veux pas qu'il sache que je suis diabétique. Si on s'entend et qu'on se marie, j'attendrais quelque temps, puis je lui dirais que le médecin vient de me dire que je suis diabétique. Je sais très bien que si les gens apprenaient que je suis diabétique, personne ne voudra se marier avec moi. Qui est ce qui va prendre pour épouse une femme malade? De toute façon, c'est ma maladie et c'est moi qui en suis atteinte, elle ne regarde que moi, je ne vois pas pourquoi j'irais en parler aux autres!»

Le malade diabétique, confronté à certaines situations, se trouve parfois dans l'obligation de se conformer à la norme sociale. À ce propos, Réda, 35 ans, sans profession, diabétique depuis cinq ans, disait :

«Lors des repas collectifs, de mariages et autres fêtes, tu es obligé de manger comme les autres sinon les gens te regardent de travers. Ne pas manger est perçu comme un manquement à l'hospitalité. Il conduit aussi au regard accusateur des gens alors je préfère manger et subir les conséquences. »

Manger apparait ainsi comme un engagement dans le groupe et un élément d'appartenance et de cohésion sociale. En effet «nous ne mangeons pas seulement pour nous nourrir, mais aussi pour des raisons cérémoniales et sociales » (Rivière, op. cit.)

On peut donc rappeler l'influence de l'environnement social immédiat sur le comportement du diabétique. Les illustrations précitées montrent bien le travail de mise en scène qu'opère le diabétique pour se prémunir du regard de l'autre et préserver ainsi sa réputation (Goffman, 1967). Elles montrent aussi comment les diabétiques se basent sur «d'autres critères d'appréciation, souvent loin du savoir des experts, liés à la vie quotidienne, à leur proximité de l'objet, à leur connaissance du milieu, à l'évaluation plus au moins juste de leurs aptitudes personnelles»(Le Breton, 1995). Le discours sur les pratiques alimentaires des diabétiques apparaît ainsi comme une grille de lecture des relations sociales selon des modalités spécifiques à l'appartenance culturelle. Cette dernière, ensemble de coutumes, de croyances et de rituels sous tend un enracinement d'habitudes alimentaires. Ces 
dernières liées aux critères hédoniques et gustatifs des aliments apparaissent aussi comme déterminantes dans les comportements des diabétiques. C'est à l'analyse de ces aspects que va être consacrée la troisième partie.

\section{Changements et résistances Les comportements alimentaires}

Le discours médical préconise de consommer des aliments en se basant sur leurs qualités diététiques. Il laisse transparaître des jugements de valeur prompts à sanctionner certains comportements comme des aberrations par rapport à une conception scientifique et idéalisée de l'aliment, réduit à ses caractéristiques nutritionnelles, économiques et, à la rigueur organoleptiques (De Garine, 1971). Son approche est d'autant plus normative qu'il s'agit d'un régime prescrit pour une maladie chronique où il est demandé aux diabétiques de se conformer à un certain nombre d'exigences basées sur des rations alimentaires reparties dans la journée. Ces dernières doivent obéir à un certain nombre de règles restrictives consistant à manger moins sucré, moins salé et moins gras. Ces recommandations qui n'envisagent le corps que dans ses dimensions biologiques et physiologiques font abstraction du sens qui est donné à l'aliment et aux fonctions hédoniques et symboliques du comportement alimentaire. Les repas constituent des moments privilégiés de la rythmique régulière de la personne et contribuent à forger des habitudes alimentaires (Rivière, op. cit.). Ces dernières, "phénomènes culturels révélateurs de l'organisation sociale et des rapports qu'une société donnée entretient avec ses membres et le milieu naturel environnant » (Bathily, 1991) ont leur impact sur le suivi du régime alimentaire.

Nacera a découvert son diabète à la suite des analyses qui sont habituellement faites après confirmation de sa grossesse. Même si elle considère que son diabète lui vient de ses parents, tous deux diabétiques, elle impute réellement son atteinte de la maladie au choc du à l'annonce de sa grossesse non désirée. Le passage au régime de Nacera n'a pas été envisagé dans la durée :

«En faisant le régime, je m'attendais à ce que je sois complètement guérie, le médecin $m$ 'avait dit qu'après la naissance du petit, le diabète allait partir, que tout redeviendrait comme avant et que j'arrêterai tout, mais finalement non, il est resté... »

La seule fois où Nacera a suivi un régime qu'elle qualifie de « strict », fût après la naissance de son enfant. La raison invoquée étant la forte peur de complications de la plaie chirurgicale de sa césarienne. Mais ce qui semble présenter un obstacle pour le maintien de son régime, ce sont ses anciennes habitudes alimentaires. À ce propos, elle disait :

"Quand on mange en famille et quand il en reste, je n'aime pas qu'il en reste. Je fais ça depuis toujours, pas seulement depuis que je suis diabétique. Je n'aime pas laisser les petites quantités de restes. Je me dis à quoi bon les garder, il vaut mieux les consommer tout de suite et ne pas les jeter, alors que normalement, on doit ramasser les restes, on les garde et quand on a faim, on réchauffe et on mange, mais moi non, je les mange tout de suite. Je fais ça depuis mon enfance, depuis que j'étais petite. C'est une habitude, je ne sais pas, c'est une habitude... une mauvaise habitude. »

Cette « mauvaise » habitude de manger les restes de repas ne s'arrête pas là. Nacera avoue ne pas concevoir cette obligation de piquer dans l'assiette comme un «poussin ». Pour elle, bien manger est synonyme de rassasiement : "J'aime manger et bien manger. J'aime sentir le ventre plein et alourdi, même si je me sens mal après. Je deviens comme une poupée sans bras ni jambes. J'aime manger à satiété, Je ne suis pas comme ce poussin qui se contente de piquer un peu de nourriture ça et là et puis c'est tout non non non, il faut que je sois 
L'ancrage social des habitudes et tradirassasiée, je ne vais pas te mentir, et le fait de manger trop... trop... (Silence). »

La manière de cuisiner les aliments semble aussi poser problème dans la mesure où les médecins préconisent de consommer les légumes cuits à la vapeur, d'éviter les fritures et l'utilisation des matières grasses, et opter plutôt pour les grillades :

Nacera : "Moi je ne veux pas préparer un menu spécialement pour moi, je te mentirais si je te disais le contraire. Je ne peux pas manger des plats cuits à la vapeur et diminuer du goût des plats. Par exemple, on nous demande de manger le poisson grillé, moi je n'aime pas le manger comme ça. J'aime le faire frire dans l'huile et j'en mange suffisamment c'est-à-dire jusqu'à ce que je n'en aie plus envie, jusqu'à ce que je sois rassasiée. Le médecin nous recommande de prendre jusqu'à quatre sardines grillées, moi je les préfère frites, je ne les aime pas cuites autrement. »

Saliha : «Je faisais cuire les légumes à la vapeur, toute sorte de légumes, les haricots, les carottes, etc. mais après je n'en pouvais plus. Je ne pouvais plus continuer toute ma vie à manger comme cela, la nourriture n'a plus de goût. Les légumes cuits de cette façon sont bien, mais à la longue, franchement ce n'est pas possible. »

Djamila : "C'est difficile de changer sa façon de manger, comme c'est difficile de changer ses habitudes. On mangeait de telle sorte après on doit manger autrement. Au début je mangeais et quand j'avais faim, je mangeais encore. Les médecins m'ont recommandée de diminuer la quantité de pain, de couscous, de gâteaux, de sucreries, de fritures et tout ce qui peut augmenter le cholestérol. D'un autre côté, il faut manger les légumes cuits à la vapeur. Même si par exemple je fais cuire les aubergines à la vapeur, pour le poisson, je n'aime pas le manger grillé. Je le fais fondre dans très peu d'huile, à feu très doux, et il a vraiment meilleur goût. Le poisson grillé, je ne l'apprécie pas. »

tions culinaires apparaît ainsi comme déterminant dans les comportements alimentaires des diabétiques. Ces derniers semblent aussi être influencés par cette préférence pour un ou plusieurs aliments fortement valorisés sur le plan symbolique (Rivière, op. cit.) à l'exemple du pain et du couscous. Ces derniers, présents quotidiennement ou occasionnellement sur les tables algériennes vont à l'encontre du régime préconisé par les médecins. Il semble difficile selon nos interlocuteurs de diminuer leur consommation. Khadîdja, 56 ans diabétique depuis vingt ans : «Le médecin nous dit de consommer une baguette de pain en la répartissant tout au long de la journée. Or cette quantité est insuffisante et je ne peux pas manger sans pain c'est comme cela ». Le pain occupe une place centrale dans l'alimentation quotidienne algérienne. En effet, les Algériens sont de grands amateurs de pain, il est présent sur toutes les tables. Sa fabrication relève du sacré car il est considéré comme une na'ma (un don de dieu). Il symbolise la communion des hommes avec le divin et les hommes entre eux (Poulain, 2002). Il accompagne presque tous les plats et principalement les ragoûts. Trempé délicatement dans la sauce, il absorbe le liquide et aide à saisir les morceaux de légumes et de viande, jouant ainsi le rôle de la fourchette (Feki, 2000). «D’un goût agréable à tous, il constitue un trait d'union entre les divers plats et fait l'unité de ceux qui le partagent... Il est symbole de sécurité, non seulement comme base de l'alimentation mais parce qu'il évoque le fruit du travail, la force investie dans la culture des céréales. La symbolique du pain est très forte. " (Rivière, 1994)

Halima déclare : «Pendant le mois de Ramadhan, je dois prendre une assiette de couscous avec du petit lait au "shour". Pour moi c'est sacré je ne peux pas m'en passer! ». Le couscous constitue bel et bien un symbole fort de la cuisine algérienne, il s'apprête de mille et une façons. Il s'accommode avec différentes sauces et est 
préparé en toutes occasions. Alimentemblème, Il tisse les liens qui unissent les membres de toute la communauté et constitue ainsi une sorte de bannière derrière laquelle, ils se retrouvent (Poulain, op. cit.).

D'autres aliments semblent incontournables, particulièrement sur la table du ftour du mois de Ramadhan. Mohamed, 52 ans est retraité de la fonction publique. Hospitalisé au moment de l'entretien, il estime que les recommandations médicales ne sont pas respectées au sein de la structure hospitalière, en revanche, il affirme :

«Une assiette de Maâkouda ${ }^{12}$ est indispensable à la table du ftour du mois de Ramadhan. Je ne peux pas concevoir la table sans. »

Saliha déclare : «Je t'avoue que je ne peux pas me passer de mon verre de limonade. Il est indispensable et personne ne pourra me l'enlever! En revanche je ne prends pas de couscous comme avant, parce qu'il me donne des douleurs au niveau du colon. Quant au régime sans sel, je diminue le sel quand je sens que ma tension est élevée. » Et Djamila, de dire : «Tu sais des fois je n'ai pas du tout de diabète, sauf quand je mange des aliments sucrés. Cette fois ma glycémie est un peu élevée, c'est à cause du berkouk ${ }^{13}$, on commence toujours le mois de Ramadhan par le sucré, pour que tout le mois Ramadhan soit aussi sucré (doux) et quand il en reste je n'aime pas le jeter et donc j'en mange et ma glycémie monte! »

Ces aliments-symboles (pain, couscous, maâkouda, berkouk, etc.) convoquent l'imaginaire de l'individu et produisent un sens qui déborde largement le cadre alimentaire. Les nourritures sont non seulement bonnes à manger mais également bonnes à penser (Strauss, 1962). En outre, ces deux dernières

12. Il s'agit de boulettes de pommes de terre frites. 13. Le berkouk (ou tajine hlou) est un plat sucré à base de fruits séchés : abricots, pruneaux, raisins secs, etc. illustrations montrent clairement comment Saliha et Djamila établissent leur rapport avec la maladie et le régime alimentaire. Ainsi, elles préfèrent être plutôt dans une logique de contrôle des symptômes, plutôt que dans une logique de contrôle de la maladie et vivre normalement leur quotidien. (Strauss et al., 1975). Cette logique «populaire » (Massé, 1995) se fonde sur la perception que le patient a de sa maladie, de l'efficacité de son traitement (Fainzang, 1997) mais aussi de son rapport aux aliments. Ces derniers acquièrent au sein des repas une mystérieuse valeur symbolique, évocatrice de réconfort, qui est l'un des facteurs de leur acceptabilité (Trémolières, 1978). Les aliments procurent du plaisir, plaisir lié à l'appréciation des mets mais aussi plaisir des repas pris en collectivité, dans la convivialité. En revanche, les restrictions et autres modifications préconisées dans le cadre du suivi du régime alimentaire remettent en cause les qualités psychosensorielles des aliments. Halima ne cache pas son goût effréné pour les fruits de saison, surtout en période d'automne. D'ailleurs, elle nous a confiés, la veille de l'entretien, avoir consommé sans retenue du raisin. Elle ajouta :

«Quand je vais aux mariages, je mange avec mes amies diabétiques, On se dit puisqu'on ne se rencontre pas souvent, alors les rares fois qu'on a l'occasion de se voir, on met le diabète de côté, on le laisse entre les mains de Dieu. On mange, on ne fait pas $d u$ tout attention. On mange ce qui nous est servi et ça ne nous fait pas de mal. Quand je suis en groupe avec la famille et les amis, je mange le plus normalement du monde et je me sens vraiment bien. Manger comme dit le médecin c'est perdre ce plaisir. La nourriture n'a plus de goût. C'est comme de la terre. »

Amina : «J'ai fait des gâteaux pour l'Aüd avec de la confiture. Il en restait sur mes doigts, j'ai résisté à la tentation. J'ai couru me rincer les mains car la maladie me l'interdit. Ainsi je ne peux pas manger les 
gâteaux que j'ai faits de mes propres mains, ensuite je ne peux pas partager ce plaisir avec mes enfants et mon mari. C'est aberrant!»

Manger s'articule avec l'affect, l'histoire personnelle du sujet et son sentiment d'appartenance (Lahlou, op . cit.). Cette affectivité s'investit fortement dans le rapport aux aliments «c'est-à-dire dans l'appétit comme dans la dégustation, dans les dégoûts comme dans les préférences, ainsi que dans le plaisir et la distraction obtenus par la consommation et la conversation » (Rivière, 1994). Les habitudes alimentaires sont des marqueurs identitaires. Elles s'incorporent dans le corps social et sous-tendent un enracinement affectif et culturel. Elles se construisent par les croyances, les pratiques alimentaires et culinaires (Kanafani-Zahar et al., 2007). Ces dernières sont étroitement liées à l'appréciation du goût, au plaisir de manger et au sens du partage.

\section{Conclusion}

Manger est un acte social complexe, « un fait social total » qui a de multiples dimensions. Il traduit une réalité profondément ancrée dans la vie quotidienne, qui ne se limite pas aux dimensions sanitaires et nutritionnelles. Le caractère collectif de l'alimentation, les habitudes et traditions culinaires associées aux critères de goût, de plaisir et de commensalité sont prédominants dans les représentations de la femme diabétique.

Cette étude a tenté de décrypter et de mettre au jour les sens attribués essentiel- lement par les femmes diabétiques aux pratiques alimentaires. Celles-ci mettent aussi en exergue le statut du diabétique dans la société patriarcale en Algérie, en dévoilant les rapports de pouvoir au sein des familles.

La dimension financière est aussi centrale pour expliquer les restrictions alimentaires opérées par les diabétiques contraints d'occulter le régime alimentaire. Le diabétique est soumis à de multiples contraintes, énoncées ci-dessus, qui modulent ses actions sans pour autant être un objet passif asservi aux forces sociales (Strauss, 1992).

La complexité de l'objet aliment mériterait une analyse plus approfondie. Il serait important de se focaliser ultérieurement sur les pratiques quotidiennes déployées aussi bien par les hommes que par les femmes, afin d'opérer une comparaison entre les deux sexes. Il s'agira de privilégier la perspective des rapports sociaux de sexe dans le champ alimentaire, en essayant de montrer notamment les différences dans les pratiques de consommation, en identifiant la logique d'imposition des hommes à l'égard des femmes. La question est de savoir si les hommes diabétiques suivraient davantage le régime alimentaire que les femmes et surtout d'en extraire les raisons profondes?

\section{Remerciements}

Je remercie vivement le Pr Mohamed Mebtoul pour ses conseils et ses encouragements, le Comité de rédaction de la revue, ainsi que les referees anonymes pour leur lecture attentive et leurs précieux et pertinents commentaires. 


\section{ANNEXE}

Tableau récapitulatif des entretiens

\begin{tabular}{c|c|c|c|c|c|c}
\hline $\begin{array}{c}\text { Prénoms } \\
\text { Données }\end{array}$ & Âge & $\begin{array}{c}\text { Situation } \\
\text { familiale }\end{array}$ & $\begin{array}{c}\text { Niveau } \\
\text { scolaire }\end{array}$ & Profession & $\begin{array}{c}\text { Ancienneté } \\
\text { du diabète }\end{array}$ & $\begin{array}{c}\text { Membres atteints } \\
\text { du diabète }\end{array}$ \\
\hline Malika & 43 & Mariée & Primaire & & 8 & Le mari \\
\hline Fatima & 34 & Célibataire & Secondaire & & 12 & Sœur et frère \\
\hline Amina & 25 & Mariée & Universitaire & Fonctionnaire & 4 & \\
\hline Zoubida & 54 & Veuve & Secondaire & Douanière & 4 & \\
\hline Hafida & 33 & Mariée & Collège & & 3 & Parents, frères sœurs \\
\hline Nacera & 43 & Mariée & Collège & & 3 & Mère et père \\
\hline Djamila & 51 & Célibataire & & Ancienne couturière & 29 & Mère et sœur \\
\hline Saliha & 39 & Mariée & Collège & & 3 & Père et frère \\
\hline Halima & 63 & Mariée & & & 5 & \\
\hline Houria & 65 & Mariée & & & 12 & \\
\hline Kheira & 56 & Mariée & & & 18 & Deux sœurs \\
\hline Khadîdja & 56 & Mariée & & & 20 & \\
\hline Fatiha & 60 & Veuve & & Retraitée & 24 & \\
\hline Mohamed & 52 & Marié & Secondaire & Retraité & 5 & \\
\hline Samir & 42 & Divorcé & Universitaire & Enseignant & 7 & \\
\hline Rachid & 35 & Célibataire & Collège & Chômeur & 5 & \\
\hline
\end{tabular}




\section{RÉFÉRENCES BIBLIOGRAPHIQUES}

Bathily A. (1991). Évolution économique et transformations des habitudes alimentaires, esquisse d'une histoire de la production vivrière dans le bassin du fleuve Sénégal des origines à l'époque contemporaine. In Igor de Garine (dir.) «les changements des habitudes et des politiques alimentaires en Afrique: aspects des sciences humaines, naturelles et sociales ». Publisud, p. 55-62.

Benabed A. (2008). Le recours à la technique de procréation médicalement assistée, étude de quelques trajectoires de couples algériens. Interrogations, revue pluridisciplinaire en sciences de l'homme et de la société, $\mathrm{n}^{\circ} 6$, la santé au prisme des sciences sociales, juin, p. 2441., http://www.revue-interrogations.org

Bihr A., Pferfferkom R.,(2000), " santé et inégalités sociales », Revue problèmes économiques, hebdo $\mathrm{n}^{\circ} 2,665, \mathrm{p} .16$.

Bouayad F.-Z. (1978). La cuisine algérienne. SNED, $439 \mathrm{p}$.

Calandre N. (2002). Alimentation, nutrition et sciences sociales, concepts, méthodes pour l'analyse des représentations et pratiques nutritionnelles des consommateurs. Mémoire de recherche pour l'obtention du DEA en économie du développement agricole, agroalimentaire et rural, sous la direction de Lucie Sirieix et Nicolas Bricas.

De Garine I. (1971). La nourriture ne sert pas qu'à s'alimenter. Revue Cérès. FAO, vol. 4, n 1 , p. 47-51.

Fainzang S. (1997). Les stratégies paradoxales. Réflexions sur l'incohérence des conduites des malades. Sciences sociales et santé, vol. 15, $\mathrm{n}^{\circ} 3$, septembre, p. 5-23.

Feki A. (2000). Cuisine tunisienne, cuisine méditerranéenne ? In colloque du GERIM, Sfax, «Alimentation et pratiques de table en méditerranée », 8-9 mars 1999, sous la direction de Yassine Essid, édition GERIM. p. 279-285.
Fassin D. (1990). Décrire. Entretien et observation. Sociétés, développement et santé. Paris, Les Éditions Ellipses, p. 87-106.

Garabuau-Moussaoui I., Palomares E. et Desjeux D., coord., 2002, « Alimentations contemporaines ", l'Harmattan. Goffman E. (1967). Les rites d'interaction. Paris, Le sens commun, Éditions de Minuit.

Hadjiat A. (2006). Diabète : le jeûne, facteur aggravant. Liberté, quotidien national d'information, p. 8.

Hamadachi K. (2009). Le diabète : la maladie des pauvres?

http://www.algerie-focus.com

Henrichs H. (2009). La surprenante diversité des aspects du diabète liés au genre. DiabetesVoice, numéro spécial, vol. 54, mai, p. 3.

Héritier-Augé F. (1985). La leçon des primitifs. In Collectif (éd.), «l'identité française ». Paris, Tierce, p. 56-65.

Kanafani-Zahar A., Mathieu S., Nizard S. (dir.) (2007). À croire et à manger : penser le lien. Paris, l'Harmattan, à croire et à manger, religions et alimentation.

Kourta D. (2006). Le diabète ausculté lors d'un congrès maghrébin, menace sur toutes les tranches d'âge. El watan, quotidien national d'information, p. 7.

Kourta D. (2006). Enquête nationale sur la santé, Les algériens mangent mal. El watan, 17 décembre, p. 6.

Kourta D. (2008). Vaste opération de dépistage au profit des patients de l'ouest. El watan, 16 décembre, p. 6.

Imbert G. (2008). Vers une étude ethno épidémiologique du diabète de type 2 et de ses complications. Société française de santé publique, $\mathrm{n}^{\circ}$ 20, février, p. 113 à 124. www. Cairn.info

Lacoste-Dujardin C., (1996), Des mères contre les femmes, Maternité et patriarcat au Maghreb, la découverte. 
Lahlou S. (1998). Penser manger. PUF, coll. Psychologie sociale, $239 \mathrm{p}$.

Le breton D. (1995). Sociologie du risque. PUF.

Massé R. (1995). Culture et santé publique, les contributions de l'anthropologie à la prévention et à la promotion de la santé. Montréal/Paris/Casablanca, Gaëtan Morin.

Mead M., Guthe C.-E. (1945). Manuel for the studies of food habits. Bulletin of national research council, national academy of sciences, $\mathrm{n}^{\circ} 111$.

Mebtoul M. (2000). La médecine comme processus social : la déconstruction d'une logique professionnelle. Santé publique et sciences sociales, $\mathrm{n}^{\circ} 6,2^{\circ}$ semestre.

Mebtoul M. (2003). Les significations attribuées à la prise en charge de deux maladies chroniques ; diabète et hypertension artérielle à Tlemcen (Algérie). Coopérations, conflits et concurrences dans le système de santé, Rennes, ENSP, p. 251-268.

Mebtoul M. (2007). Quand les habitudes alimentaires mettent en défaut les normes médicales. El Joumhouria, p. 19.

Poulain J.-P. (2002). Sociologies de l'alimentation. Les mangeurs et l'espace social alimentaire. Paris, PUF, coll. Sciences sociales et sociétés, $286 \mathrm{p}$.
Régnier F. (2009). Obésité, goûts et consommation. Intégration des normes d'alimentation et appartenance sociale. Revue française de sociologie, 50-4, p. 747-773.

Rivière C. (1994). Les rituels du manger. Revue Prévenir, $\mathrm{n}^{\circ}$ 26, p. 7-29.

Rivière C. (1995). Les rites profanes. PUF. Strauss A. (1992). La trame de la négociation, sociologie qualitative et interactionnisme. Textes réunis et présentés par I. Baszanger, Paris, L'Harmattan.

Strauss A., Corbin J., Fagerhaugh S., Glaser B.-G., Maines D., Suczeck B., Wiener C. (1975). Chronic illness and the quality of life. Saint-Louis, CV Mosby Co.

Strauss C.-L. (1962). Le totémisme aujourd'hui. Paris, PUF.

Suremain (de) C.-H., Katz E. (2008). Introduction : modèles alimentaires et recompositions sociales en Amérique latine. Anthropology of Food, http:/aof.revues.org

Transition And Health Impact in North Africa (TAHINA) (2007). Contrat $n^{\circ} I$ CA3- CT-2002-10011. Document PDF, http://www.mpl.ird.fr/tahina/index.htm, $305 \mathrm{p}$.

Trémolières J. (1978). Partager le pain. Éditions Robert Laffont. 